\title{
Optimising acquisition parameters for myocardial T2 mapping using T2-prep at $3 \mathrm{~T}$
}

\author{
EM Tunnicliffe ${ }^{1,2^{*}}$, MD Robson $^{1}$ \\ From 16th Annual SCMR Scientific Sessions \\ San Francisco, CA, USA. 31 January - 3 February 2013
}

\section{Background}

T2 mapping using T2-prepared SSFP at 1.5T has been shown to be sensitive to oedema in acute myocardial infarction[1]. At 3T, other studies have addressed the problem of the T2 prep module's sensitivity to the increased B1 inhomogeneity[2]. However, a second problem is that the lengthened $\mathrm{T} 1$ at $3 \mathrm{~T}$ can reduce the contrast between normal and oedematous myocardium, as well as introducing heart rate variability in measured $\mathrm{T} 2$. We set out to develop a protocol which maximised the difference between measured $\mathrm{T} 2$ in normal and oedematous tissue, while minimising the heart-rate dependence of the measured $\mathrm{T} 2$.

\section{Methods}

Two gel phantoms (agarose and $\mathrm{NiCl}_{2}$ ) were constructed with relaxation times close to normal myocardium $(\mathrm{N})$ and oedematous myocardium $(\mathrm{O})[1]$,3 . These relaxation times were measured at $3 \mathrm{~T}$ (Siemens Verio) using ShMOLLI[3] and a multi-echo spin echo sequence.

Next, a T2-prepared T2 mapping sequence (Siemens WIP 447) was used, with the default 1.5T protocol[1] varied as follows: GRE readout, flip angle $=5^{\circ}, 9^{\circ}, 18^{\circ}$; SSFP readout, flip angle $=20^{\circ}, 35^{\circ}, 50^{\circ}$; Linear and centric k-space ordering, and with the order of the $\mathrm{T} 2$ prep module echo times permuted $(0,32,55 \mathrm{~ms} ; 0,55,32 \mathrm{~ms}$ etc.). Each protocol variant was run with heart periods of $600 \mathrm{~ms}, 1000 \mathrm{~ms}$ and $1200 \mathrm{~ms}$ (heart rates of 50, 60 and $100 \mathrm{bpm}$ ), for a total of 216 scans.

The reconstructed T2 maps were analysed using Matlab (Natick, MA). Each protocol was evaluated for its ability to distinguish between normal and oedematous myocardium by comparing the difference between the measured $\mathrm{T} 2$, averaged over a ROI covering the body of the $\mathrm{N}$ and $\mathrm{O}$ phantoms. The standard deviation of measured T2 for each protocol across the three heart rates was used as a measure of heart-rate dependence. A $\mathrm{t}$-test was used to determine whether the difference in T2 was statistically significant over all heart rates, and to rank the protocols, with the lowest p-value protocol providing the best discrimination between the $\mathrm{T} 2$ of the two phantoms.

\section{Results}

The measured reference relaxation times for the two phantoms were N: T1/T2=1152/53ms; O: T1/T2=1302/ $59 \mathrm{~ms}$. Apparently minor changes in the acquisition protocol yield wildly different $\mathrm{T} 2$ values using these methods (Figure 1a). No protocol provided measurements of T2 within $3 \mathrm{~ms}$ for both phantoms over all heart rates and many approaches demonstrated considerable bias from the true $\mathrm{T} 2$ value.

Selected pertinent protocol details and numeric results are summarised in Table 1. Figure $1 \mathrm{~b}$ shows the detection of oedema in a patient using protocol $\mathrm{D}$.

\section{Conclusions}

The sensitivity of myocardial T2 mapping at $3 \mathrm{~T}$ can be significantly improved by optimising acquisition parameters. Based on this phantom study, we use a centrically ordered GRE readout with a flip angle of $18^{\circ}$ and a T2-prep order of 32-55-0ms. 

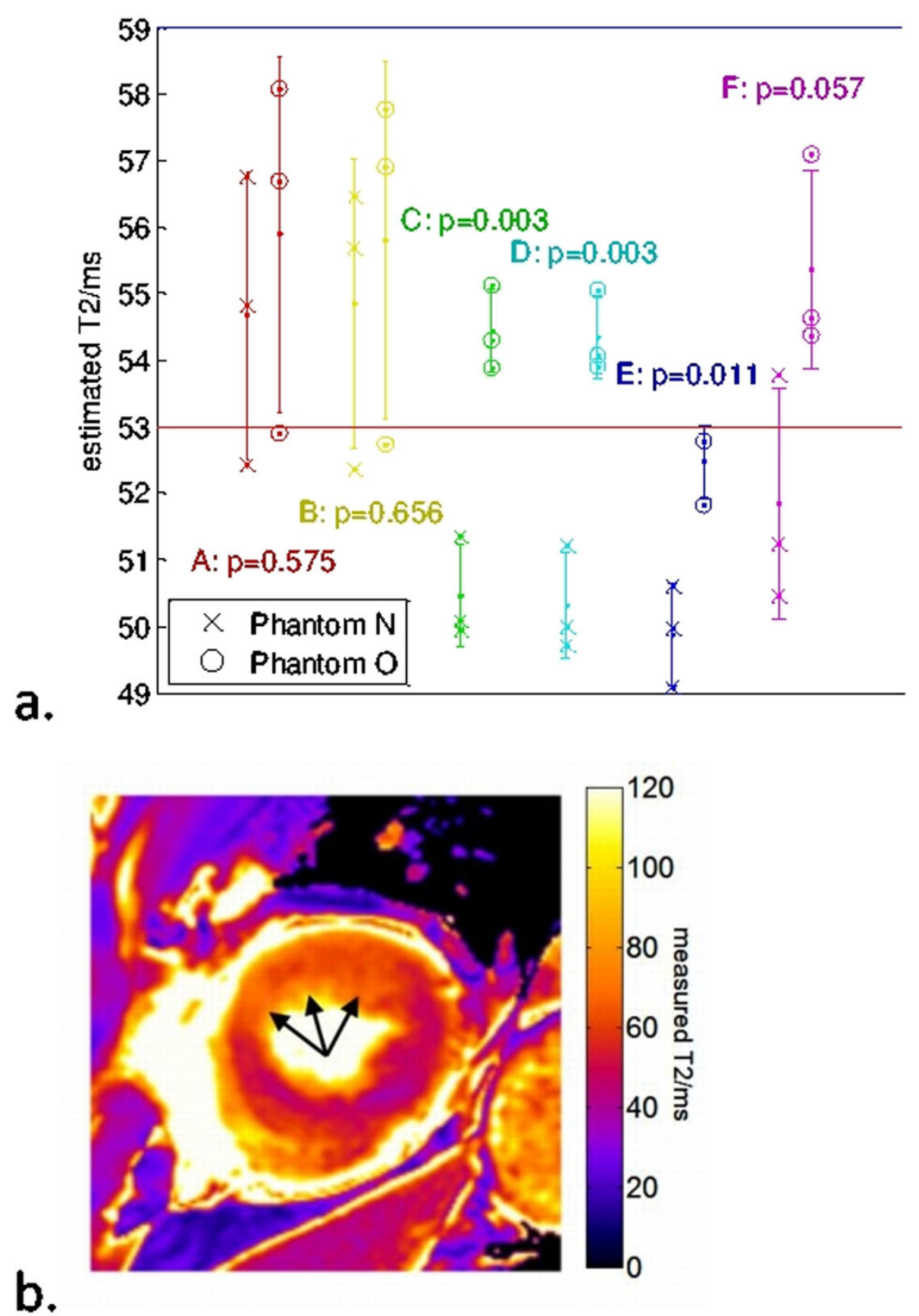

Figure 1 a. Measured T2 on phantoms with relaxation times close to normal $(\mathrm{N})$ and oedematous $(\mathrm{O})$ myocardium with a subset of the protocols tested. Red and blue dotted lines show the true $\mathrm{T} 2$ of phantoms $\mathrm{N}$ and $\mathrm{O}$ respectively. The three points for each phantom and protocol represent the three heart rates (with higher heart rates leading to longer measured T2 values) and error bars show the mean \pm s.d. over heart rate. Protocol details are in Table 1. b. Discrimination of oedematous (arrowed) and remote myocardium in acute myocardial infarction using protocol D. 
Table 1 Protocols for comparison are: the default 1.5T protocol, and default 1.5T protocol with GRE readout (A\&B); the three best protocols (lowest p-value, i.e. best discrimination between measured T2), two GRE (C\&D) and one SSFP (E); the best protocol with an SSFP readout substituted for GRE (F).

\begin{tabular}{|c|c|c|c|c|c|}
\hline $\begin{array}{l}\text { Protocol (Figure } \\
\text { 1a) }\end{array}$ & Protocol details & $\begin{array}{l}\text { Mean difference in } \\
\mathrm{T} 2 / \mathrm{ms}\end{array}$ & $\begin{array}{l}\text { Quadrature-combined s.d. over all } \\
\text { heart rates }\end{array}$ & $\begin{array}{l}\text { t-test } p \text { - } \\
\text { value }\end{array}$ & Comment \\
\hline A & $\begin{array}{l}\text { SSFP, } 50^{\circ} \text {, linear, 0-32- } \\
55 \mathrm{~ms}\end{array}$ & 1.2 & 3.5 & 0.575 & 1.5T default protocol[1] \\
\hline B & $\begin{array}{l}\text { GRE, } 9^{\circ}, \text { linear, 0-32- } \\
55 \mathrm{~ms}\end{array}$ & 1.0 & 3.5 & 0.656 & $\begin{array}{l}1.5 T \text { default protocol with GRE } \\
\text { readout }\end{array}$ \\
\hline C & $\begin{array}{l}\text { GRE, } 18^{\circ} \text {, centric, 32-0- } \\
55 \mathrm{~ms}\end{array}$ & 4.0 & 1.0 & 0.003 & Lowest p-value \\
\hline D & $\begin{array}{l}\text { GRE, } 18^{\circ} \text {, centric, 32- } \\
55-0 \mathrm{~ms}\end{array}$ & 4.0 & 1.0 & 0.003 & Lowest p-value \\
\hline $\mathrm{E}$ & $\begin{array}{c}\text { SSFP, } 20^{\circ} \text {, centric, } 0-55- \\
32 \mathrm{~ms}\end{array}$ & 2.6 & 0.95 & 0.011 & $\begin{array}{l}\text { Lowest p-value with SSFP } \\
\text { readout }\end{array}$ \\
\hline $\mathrm{F}$ & $\begin{array}{c}\text { SSFP, } 50^{\circ} \text {, centric, } 32-0- \\
55 \mathrm{~ms}\end{array}$ & 3.5 & 2.3 & 0.057 & $\begin{array}{l}\text { Best protocol but with SSFP } \\
\text { readout }\end{array}$ \\
\hline
\end{tabular}

\section{Funding}

We thank the NIHR Oxford Biomedical Research Centre and UK Department of Health for grant funding.

Author details

${ }^{1}$ OCMR, Radcliffe Department of Medicine, University of Oxford, Oxford, UK.

${ }^{2} \mathrm{AVIC}$, Radcliffe Department of Medicine, University of Oxford, Oxford, UK.

Published: 30 January 2013

\section{References}

1. Giri, et al: JCMR 2009, 11:56.

2. Jenista, et al: JCMR 2011, 13(S1):P2.

3. Piechnik, et al: JCMR 2010, 12:69.

\section{doi:10.1186/1532-429X-15-S1-W10}

Cite this article as: Tunnicliffe and Robson: Optimising acquisition parameters for myocardial T2 mapping using T2-prep at 3T. Journal of Cardiovascular Magnetic Resonance 2013 15(Suppl 1):W10.

Submit your next manuscript to BioMed Central and take full advantage of:

- Convenient online submission

- Thorough peer review

- No space constraints or color figure charges

- Immediate publication on acceptance

- Inclusion in PubMed, CAS, Scopus and Google Scholar

- Research which is freely available for redistribution 